DSF-21/2005

July 2005

\title{
On the Absence of Continuous Symmetries for Noncommutative 3-Spheres
}

\author{
Fedele Lizzi $^{a}$, Allen Stern $^{a, b}$ And Patrizia Vitale ${ }^{a}$ \\ a) Dip. Scienze Fisiche, Università di Napoli Federico II \\ and INFN Sez. di Napoli, \\ Compl. Univ. Monte S. Angelo, Napoli, 80126, Italy \\ b) Department of Physics, University of Alabama, \\ Tuscaloosa, Alabama 35487, USA \\ fedele.lizzi@na.infn.it, patrizia.vitale@na.infn.it, astern@bama.ua.edu
}

\begin{abstract}
A large class of noncommutative spherical manifolds was obtained recently from cohomology considerations. A one-parameter family of twisted 3-spheres was discovered by Connes and Landi, and later generalized to a three-parameter family by Connes and Dubois-Violette. The spheres of Connes and Landi were shown to be homogeneous spaces for certain compact quantum groups. Here we investigate whether this property can be extended to the noncommutative three-spheres of Connes and Dubois-Violette. Upon restricting to quantum groups which are continuous deformations of $\operatorname{Spin}(4)$ and $S O(4)$ with standard co-actions, our results suggest that this is not the case.
\end{abstract}




\section{Introduction}

The recent interest in noncommutative geometry [1] has led to an on-going search for non-trivial examples of noncommutative spaces. Noncommutative generalizations of spheres in various dimensions are known (for a review see [2]), but many of these suffer from a drop in dimensions. Actually, the dimension of a noncommutative space is not uniquely defined. One choice which uses concepts which are natural in noncommutative geometry is the Hochschild dimension. It plays an important role for the three-parameter family of deformations of the sphere $S^{3}$ introduced by Connes and Dubois-Violette [3], which generalizes the one-parameter family discovered previously by Connes and Landi 4]. The Hochschild dimension of the corresponding algebra remains constant (and equal to three) for the deformation. A generalization to higher dimensions is possible for the one-parameter subset, the so-called twisted spheres. This particular subset has another important characteristic. It has been shown [5, 6] that the spheres in the one parameter subset carry a coaction of the multiparametric quantum orthogonal groups $S O_{\theta}(n+1)$ [7; i.e., they are homogeneous spaces of quantum groups. The aim of this paper is to investigate the possibility of defining a group coaction for the three-parameter 3-spheres as well.

The algebra of the three-parameter spheres $S_{u}^{3}[3$ is generated by hermitean operators $\hat{x}_{\mu}, \mu=0,1,2,3$, subject to

$$
\hat{x}_{\mu} \hat{x}_{\mu}=11,
$$

where 11 is the unit operator, and quadratic commutation relations

$$
\left[\hat{x}_{\mu}, \hat{x}_{\nu}\right]=i \hat{E}_{\mu \nu, \rho \sigma} \hat{x}_{\rho} \hat{x}_{\sigma}
$$

The constant coefficients $\hat{E}_{\mu \nu, \rho \sigma}$ are expressed in terms of four angles $\phi_{\mu}$,

$$
\hat{E}_{\mu \nu, \rho \sigma}=\epsilon_{\mu \nu \rho \sigma} \frac{\sin \left(\phi_{\rho}-\phi_{\sigma}\right)}{\cos \left(\phi_{\mu}-\phi_{\nu}\right)}, \quad \text { no sum on repeated indices }
$$

$\hat{E}_{\mu \nu, \rho \sigma}$ is antisymmetric in the first two indices and symmetric in the last two indices. These commutation relations hold provided no two angles differ by $\pi / 2$. Because they depend only on the difference of angles there are three independent deformation parameters, and so one angle, say $\phi_{0}$, can be set to zero. The Connes-Landi case has two of the remaining angles equal with the third zero; e.g., $\phi_{1}=\phi_{2}=\frac{\phi}{2}$ and $\phi_{3}=0$ [4].

In the search for continuous symmetries, we shall consider linear, as well as spinor, transformations. When all angles are set to zero we require that the symmetries reduce to $S O(4)$ and Spin(4) transformations, respectively. On the other hand, we cannot get Lie group transformations when any of the independent parameters are nonvanishing, 
since (1.2) would in general not be preserved. If they exist, such symmetries should correspond to quantum group transformations. As the Connes- Dubois-Violette three-spheres are three-parameter deformations of the sphere, their symmetries should correspond to multiparametric deformations of $S O(4)$ and $\operatorname{Spin}(4)$. Multiparametric deformations of orthogonal groups [7, 8] can be obtained from the standard one-parameter quantum group by applying a twist $F$, which depends on additional parameters $q_{a b}$, to the quantum $R$ matrix. The twist is required to be a specific function in the universal enveloping algebra of the Lie group under consideration. Under these assumptions the quantum deformation becomes at most two-parametric in the case of $S O(4)$. Therefore these kind of q-groups cannot be be associated with symmetries for the full three-parameter family of noncommutative spheres. Alternatively, it is possible that there exist symmetries associated with quantum groups which are not deformable to Lie-groups. Here, however, our primary focus will be on continuous deformations of $\operatorname{Spin}(4)$ and $S O(4)$. We are then justified in looking at the limit of small angles where the search for continuous symmetries is considerably simplified. This is the commutative limit, where the noncommutative sphere goes to $S^{3}$, with $\hat{x}_{\mu}$ going to real commuting coordinates $x_{\mu}$, $x_{\mu} x_{\mu}=1$, and the noncommutativity gets replaced by a nontrivial Poisson structure on $S^{3}$. The commutative limit of the quantum group associated with a continuous symmetry, if it exists, is a Lie-Poisson group [9], a Lie group with a Poisson bracket on the group manifold which is compatible with the group multiplication.

The search for Lie-Poisson symmetry in the case of the commutative limit of the Connes and Dubois-Violette spheres is carried in sections 2 and 3. The Poisson brackets are recovered from (1.2) in the limit of small angles $\phi_{\mu} \rightarrow \epsilon_{\mu}$

$$
\left\{x_{\mu}, x_{\nu}\right\}=E_{\mu \nu, \rho \sigma} x_{\rho} x_{\sigma}
$$

where

$$
E_{\mu \nu, \rho \sigma}=\epsilon_{\mu \nu \rho \sigma}\left(\epsilon_{\rho}-\epsilon_{\sigma}\right), \quad \text { no sum on repeated indices }
$$

Our approach is to express the Poisson brackets on $S^{3}$ in terms of a constant matrix, with the intention of utilizing it as a classical R-matrix for a Lie-Poisson group. In section 3 we consider Spin(4) transformations. For this one expresses the coordinates in terms of an $S U(2)$ matrix $u$, with the $\operatorname{Spin}(4)=S U(2) \times S U(2)$ transformation given by

$$
u \rightarrow u^{\prime}=g u h^{-1}
$$

where $g$ and $h$ are independent elements of $S U(2)$. The problem is then to find a Poisson structure on $\operatorname{Spin}(4)$ which is compatible with the Poisson algebra on $S^{3}$. This means that both group multiplication and the action on $S^{3}$ are Poisson maps. The former property defines the Lie-Poisson group. Expressing the Poisson brackets on the group 
in terms of a classical $R$-matrix, which by definition satisfies the classical Yang-Baxter equations, insures that the Jacobi identity is satisfied. If such a classical $R$-matrix is found, the group is a Lie-Poisson group, and the space under consideration would be a homogeneous space of the Lie-Poisson group. In section 2 we find a consistent classical $R$-matrix only in the Connes-Landi limit, and thus we only get a Poisson map of Spin(4) in this case. In section 3 we get the same result for $S O(4)$. We assume the usual linear $S O(4)$ transformations

$$
x_{\mu} \rightarrow x^{\prime}{ }_{\mu}=M_{\mu \nu} x_{\nu}
$$

$M_{\mu \nu}$ being $S O(4)$ matrix elements. A candidate for the Poisson brackets of $M_{\mu \nu}$ can be written in terms of a constant $16 \times 16$ matrix, but the latter only defines a classical $R$-matrix, i.e., satisfies the classical Yang-Baxter equations, in the Connes-Landi limit.

After ruling out symmetries associated with continuous deformations of $\operatorname{Spin}(4)$ and $S O(4)$, there still is the possibility of symmetries at certain finite angles $\phi_{\mu}$. This case is more difficult to analyze since it involves going to the full noncommutative theory. In section 4 we investigate the full noncommutative theory and search symmetries associated with spinor-type transformations. We express the algebra for the twisted three-sphere in terms of a possible quantum R-matrix. The quantum Yang-Baxter equations should be satisfied for the corresponding quantum group algebra to be co-associative. This cannot be true for arbitrary continuous deformations of the commutative sphere, since in the limit of small angles we recover the system of section 2 . In section 4 we further find no finite values of $\phi_{\mu}$, other than those in the Connes-Landi limit, for which the candidate R-matrix satisfies the quantum Yang-Baxter equations.

In section 5 we Wick rotate the system of Connes and Dubois-Violette, leading to "noncommutative hyperboloids" in Minkowski space, and repeat some of the previous analysis in search of quantum deformations of the Lorentz group which have twisted hyperboloids as homogeneous spaces. As before the search is only successful for a one parameter subset of hyperboloids, namely being the Wick rotation of Connes-Landi spheres.

In section 6 we give concluding remarks and discuss the prospects for a more exhaustive study of the full noncommutative theory.

\section{Poisson action of $\operatorname{Spin}(4)$}

As $u$ appearing in (1.6) is in the defining representation of $S U(2)$ it can be expressed in terms of the coordinates according to

$$
u=x_{\mu} \tau^{\mu},
$$


where $\tau^{0}$ is the $2 \times 2$ identity matrix $\tau^{0}=1_{2 \times 2}$ and $\tau^{i}$ are $i$ times the Pauli matrices, $\tau^{i}=i \sigma^{i}, i=1,2,3 . \tau^{\mu}$ satisfy

$$
\frac{1}{2} \operatorname{Tr}\left(\tau^{\mu} \tau^{\nu}\right)=\eta^{\mu \nu}=\operatorname{diag}(1,-1,-1,-1)
$$

$u$ has real trace and the traceless part is anti-hermitean. Hermitean conjugation corresponds to a parity transformation.

We next show that the Poisson brackets (1.4) can be written in the form

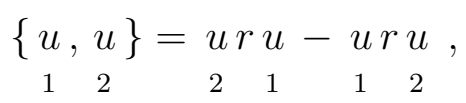

where by $u$ and $u$ is meant $u \otimes 1$ and $1 \otimes u$ respectively, and $r$ is a $4 \times 4$ matrix which we need to determine*. From

$$
u u^{\dagger}=u^{\dagger} u=1_{2 \times 2},
$$

$r$ must be hermitean. From the antisymmetry of the Poisson bracket $r$ should be invariant under exchange of the two tensor product spaces; i.e., $r=r=r$. Finally in order to recover the Poisson brackets (1.4) from (2.3) $r$ should satisfy

$$
\tau_{2}^{\rho} r \tau^{\sigma}-\tau^{\rho} r \tau^{\sigma}+\tau^{\sigma} r \tau^{\rho}-\tau^{\sigma} r \tau^{\rho}=2 E_{\mu \nu, \rho \sigma} \tau^{\mu} \tau^{\nu}
$$

The solution (up to a term proportional to the identity matrix $\mathbb{1}_{4 \times 4}=\tau^{0} \times \tau^{0}$ ) is

$$
r=\kappa_{1} \tau^{1} \otimes \tau^{1}+\kappa_{2} \tau^{2} \otimes \tau^{2}+\kappa_{3} \tau^{3} \otimes \tau^{3}
$$

where $\kappa_{i}$ are given by

$$
\begin{aligned}
& \kappa_{1}=\frac{1}{2}\left(-\epsilon_{0}-\epsilon_{1}+\epsilon_{2}+\epsilon_{3}\right) \\
& \kappa_{2}=\frac{1}{2}\left(-\epsilon_{0}+\epsilon_{1}-\epsilon_{2}+\epsilon_{3}\right) \\
& \kappa_{3}=\frac{1}{2}\left(-\epsilon_{0}+\epsilon_{1}+\epsilon_{2}-\epsilon_{3}\right)
\end{aligned}
$$

\footnotetext{
${ }^{*}$ For a more general starting ansatz see the end of this section.
} 
The standard action of $\operatorname{Spin}(4)$ on $S^{3}$ is (1.6), where $g$ and $h$ are independent elements of $S U(2)$ in the defining representation. The Poisson algebra (2.3) is not preserved under this action. Instead, (2.3) goes to

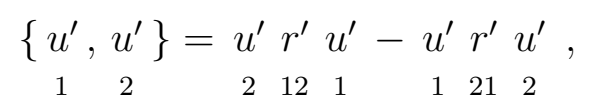

where

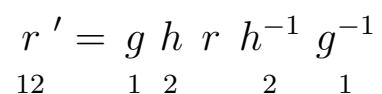

On the other hand, if we can consistently assign the following Poisson structure to $\operatorname{Spin}(4)$ :

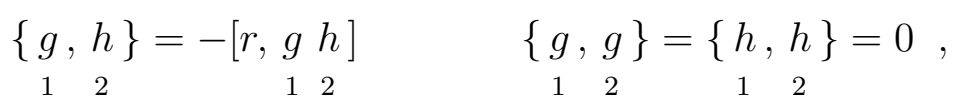

the brackets (2.3) are invariant in the sense that (1.6) is a Poisson map. For these Poisson brackets to be consistent we need that they are antisymmetric and satisfy the Jacobi identity. This means that $r$ should satisfy the classical Yang-Baxter equations

$$
[r, r+r]+[r, r]=I^{\prime}
$$

where $I^{\prime}$ is an adjoint invariant for Spin(4). Note that the classical Yang-Baxter equations did not have to be satisfied for (2.3) to be consistent with the Jacobi identity. It is easily seen that the classical Yang-Baxter and hence the Jacobi identity for (2.10) are satisfied when all $\kappa_{i}$ but one vanish. This corresponds to two angles being equal while the third is zero, i.e. the Connes-Landi case. Moreover, the Yang-Baxter equations and Jacobi identity are only satisfied in this case, and thus only then do $g$ and $h$ generate a Lie-Poisson group. In that case we can introduce spinors $\psi=\left(\begin{array}{l}\psi_{1} \\ \psi_{2}\end{array}\right)$ and $\bar{\psi}=\left(\begin{array}{ll}\bar{\psi}_{1} & \bar{\psi}_{2}\end{array}\right)$ with Poisson brackets

$$
\left\{\psi_{a}, \bar{\psi}_{b}\right\}=r_{a d, c b} \psi_{c} \bar{\psi}_{d}
$$

for which

$$
\psi \rightarrow \psi^{\prime}=g \psi, \quad \bar{\psi} \rightarrow \bar{\psi}^{\prime}=\bar{\psi} h^{-1}
$$

will be a Poisson map. Then the Poisson algebra for $\psi \bar{\psi}$ is identical to that for $u$ in (2.3). 
Concerning (2.3), we could start with the most general ansatz which is linear in both $u$ and $u$ :

12

$$
\{u, u\}=r^{(1)} u \underset{2}{u} \begin{gathered}
u \\
1
\end{gathered} 2_{2} u r^{(2)}-u r^{(3)} u-u r^{(4)} u
$$

where we have introduced four $4 \times 4$ matrices $r^{(A)}, A=1,2,3,4$. Now (1.6) is a Poisson map when (2.10) is generalized to

$$
\begin{aligned}
& \underset{1}{\{g}, \underset{2}{g}\}=\left[r^{(1)}, g_{1} g_{2}\right] \\
& \{h, h\}=\left[r^{(2)}, h h\right] \\
& \left.\underset{1{ }_{2}}{g, h}\right\}=\left[r^{(3)}, g_{1}{ }_{2} h\right]
\end{aligned}
$$

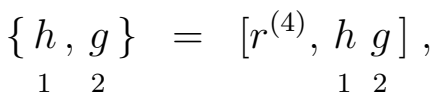

The matrices $r^{(A)}, A=1,2,3,4$, are not fully determined from the Poisson brackets (1.4) of the coordinates. The ambiguity can be fixed once one imposes the requirements that the Poisson brackets for the matrix elements of $g$ and $h$ be antisymmetric and consistent with $\operatorname{det} g=\operatorname{det} h=1$. However, it can be shown, that then the brackets (2.14) and (2.15) collapse to (2.3) and (2.10), and so the previous conclusions apply.

\section{Poisson action of $S O(4)$}

From the $\operatorname{Spin}(4)$ transformations (1.6) we can construct the corresponding $S O(4)$ transformations (1.7), and since the former defines a Poisson map in the Connes-Landi case so does the latter. In that case $S O(4)$ matrix elements $M_{\mu \nu}$ are expressed as quadratic functions of group elements $g$ and $h$

$$
M_{\mu \nu}(g, h)=\frac{1}{2} \operatorname{Tr}\left(\tau_{\mu} g \tau^{\nu} h^{-1}\right),
$$

where the indices on $\tau$ are raised and lowered with the Minkowski metric (2.2). More generally, if we don't make assumptions like (3.1), it may be possible to find a Poisson 
map of a group even when no Poisson map is induced by its covering group. However, we find that not to be the case for $S O(4)$ acting on the noncommutative 3-sphere, i.e., like Spin(4), $S O(4)$ has a Poisson action only in the Connes-Landi case.

The Poisson brackets (1.4) are not preserved under the action (1.7) of $S O(4)$. Rather they are transformed to

$$
\left\{x_{\mu}^{\prime}, x_{\nu}^{\prime}\right\}=E_{\mu \nu, \rho \sigma}^{\prime} x_{\rho}^{\prime} x_{\sigma}^{\prime}
$$

where

$$
E_{\mu \nu, \rho \sigma}^{\prime}=M_{\mu \alpha} M_{\nu \beta} M_{\rho \gamma} M_{\sigma \delta} E_{\alpha \beta, \gamma \delta}
$$

On the other hand, it may be possible to have Poisson brackets on $S O(4)$ which define a Lie-Poisson group and make (1.7) a Poisson map. The Poisson brackets are required to satisfy

$$
\left(\left\{M_{\mu \gamma}, M_{\nu \delta}\right\}-E_{\mu \nu, \rho \sigma} M_{\rho \gamma} M_{\sigma \delta}+M_{\nu \sigma} M_{\mu \rho} E_{\rho \sigma, \gamma \delta}\right) x_{\gamma} x_{\delta}=0,
$$

in addition to antisymmetry and the Jacobi identity. (3.4) is solved by

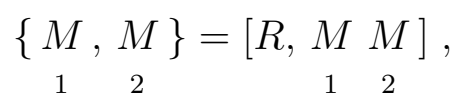

where

$$
R_{\mu \nu, \rho \sigma}=E_{\mu \nu, \rho \sigma}+A_{\mu \nu, \rho \sigma},
$$

and $A$ is antisymmetric in the last two indices, $A_{\mu \nu, \rho \sigma}=-A_{\mu \nu, \sigma \rho}$. From the requirement that $M$ is orthogonal it follows that $R$ should be a symmetric matrix $R_{\mu \nu, \rho \sigma}=R_{\rho \sigma, \mu \nu}$. This then fixes $A_{\mu \nu, \rho \sigma}=E_{\rho \sigma, \mu \nu}$, and hence $R$ becomes

$$
R_{\mu \nu, \rho \sigma}=\epsilon_{\mu \nu \rho \sigma}\left(\epsilon_{\mu}-\epsilon_{\nu}+\epsilon_{\rho}-\epsilon_{\sigma}\right)
$$

Antisymmetry of the Poisson bracket follows since $R$ is antisymmetric under exchange of the tensor product spaces. $R$ can be expressed in terms of tensor products of $S O(4)$ generators $J_{i}$ and $K_{i}, i=1,2,3$, written in the defining representation:

$$
\begin{aligned}
& J_{1}=\frac{1}{2 \sqrt{2}}\left(\begin{array}{cccc}
0 & 1 & 0 & 1 \\
-1 & 0 & 1 & 0 \\
0 & -1 & 0 & 1 \\
-1 & 0 & -1 & 0
\end{array}\right) \quad K_{1}=\frac{1}{2 \sqrt{2}}\left(\begin{array}{cccc}
0 & -1 & 1 & 0 \\
1 & 0 & 0 & 1 \\
-1 & 0 & 0 & 1 \\
0 & -1 & -1 & 0
\end{array}\right) \\
& J_{2}=\frac{1}{2 \sqrt{2}}\left(\begin{array}{cccc}
0 & 1 & 0 & -1 \\
-1 & 0 & -1 & 0 \\
0 & 1 & 0 & 1 \\
1 & 0 & -1 & 0
\end{array}\right) \quad K_{2}=\frac{1}{2 \sqrt{2}}\left(\begin{array}{cccc}
0 & 1 & 1 & 0 \\
-1 & 0 & 0 & 1 \\
-1 & 0 & 0 & -1 \\
0 & -1 & 1 & 0
\end{array}\right)
\end{aligned}
$$




$$
J_{3}=\frac{1}{2}\left(\begin{array}{cccc}
0 & 0 & -1 & 0 \\
0 & 0 & 0 & 1 \\
1 & 0 & 0 & 0 \\
0 & -1 & 0 & 0
\end{array}\right) \quad K_{3}=\frac{1}{2}\left(\begin{array}{cccc}
0 & 0 & 0 & -1 \\
0 & 0 & 1 & 0 \\
0 & -1 & 0 & 0 \\
1 & 0 & 0 & 0
\end{array}\right)
$$

They satisfy

$$
\begin{aligned}
{\left[J_{i}, J_{j}\right] } & =\epsilon_{i j k} J_{k} \\
{\left[K_{i}, K_{j}\right] } & =\epsilon_{i j k} K_{k} \\
{\left[J_{i}, K_{j}\right] } & =0
\end{aligned}
$$

Defining $J_{ \pm}=\sqrt{2}\left(J_{1} \pm J_{2}\right)$ and $K_{ \pm}=\sqrt{2}\left(K_{1} \pm K_{2}\right), R$ can be written $R=2 \kappa_{1}\left(J_{-} \otimes K_{3}-K_{3} \otimes J_{-}\right)+2 \kappa_{2}\left(K_{+} \otimes J_{3}-J_{3} \otimes K_{+}\right)+\kappa_{3}\left(K_{-} \otimes J_{+}-J_{+} \otimes K_{-}\right)$,

where $\kappa_{i}$ are again given by (2.7). Finally we need to check that (3.5) satisfies the Jacobi identity, or equivalently that $R$ satisfies the classical Yang-Baxter equations:

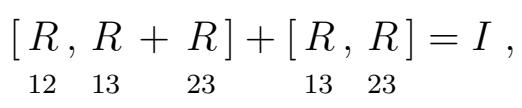

where $I$ is an adjoint invariant for $S O(4)$. From $\left[J_{i}, K_{j}\right]=0$, it is easily seen that (3.11) is satisfied when all $\kappa_{i}$ but one vanish. Thus the Jacobi identity is satisfied when two angles are equal and the third is zero. Just as with Spin(4), the Yang-Baxter equations and Jacobi identity are only satisfied in this case, and thus $\left[M_{\mu \nu}\right]$ generate a Lie-Poisson group only in this case. It can be checked that the results in this case agree with the lowest order commutation relations in [6].

Above we have argued that we can consistently define a Poisson algebra for the $S O(4)$ matrices only in the Connes-Landi case. This algebra is obtainable from the Poisson algebra (2.10) on Spin(4) using (3.11):

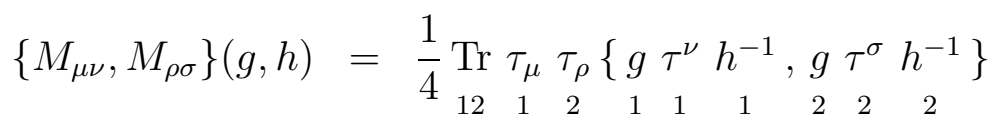

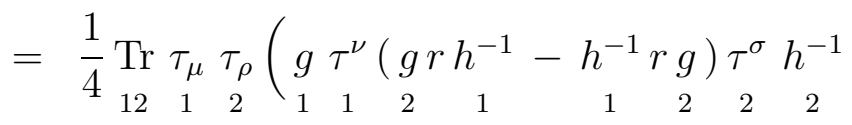

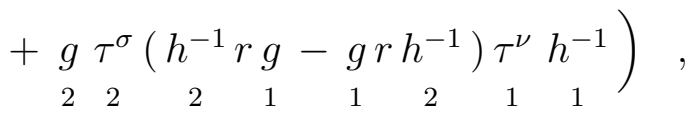


where $\operatorname{Tr}$ means a trace over both tensor product spaces. We then recover the expression 12

(3.5) with $R$ given by

$$
R_{\mu \nu, \rho \sigma}=\frac{1}{4} \operatorname{Tr}_{12}\left[\tau_{\rho} \otimes \tau^{\nu}, \tau^{\mu} \otimes \tau_{\sigma}\right] r
$$

After substituting in the general expression for $r$ given in (2.6) one then gets (3.7).

\section{Quantum Spin(4) Transformation}

Here we generalize to the full noncommutative theory with the goal of searching for symmetries of the noncommutative three-sphere occurring at finite angles $\phi_{\mu}$. For simplicity we restrict to spinor-type transformations thereby generalizing the discussion of Section 2.

We begin by replacing the $2 \times 2$ matrix $u$ by another $2 \times 2$ matrix $\hat{u}$, the latter having noncommuting matrix elements. The property of unitarity

$$
\hat{u} \hat{u}^{\dagger}=\hat{u}^{\dagger} \hat{u}=1_{2 \times 2},
$$

can be maintained although $\hat{u}$ does not have to have unit determinant. For this (2.1) should be generalized to

$$
\hat{u}=\hat{x}_{\mu} e^{i \phi_{\mu}} \tau^{\mu} .
$$

The sum over $\mu=0,1,2,3$ is assumed. The unitarity condition was shown [3] to be consistent with the commutation relations (1.2). We next show that the commutation relations can be expressed as

$$
\begin{aligned}
& \hat{u} \hat{r} \hat{u}=\hat{u} \hat{r} \hat{u} \\
& \begin{array}{llll}
1 & 2 & 2 & 1
\end{array}
\end{aligned}
$$

for some $4 \times 4$ matrix $\hat{r}$. These relations are invariant under interchange of the two tensor product spaces provided $\hat{r}$ is invariant under interchange of the two tensor product

spaces; i.e., $\hat{r}=\underset{12}{\hat{r}}=\underset{21}{\hat{r}}$. In the limit of small angles $\phi_{\mu} \rightarrow \epsilon_{\mu}, \hat{r}$ should reduce to $1_{4 \times 4}+i r$, with $r$ given in (2.6), for then the commutator of $\hat{u}$ with $\hat{u}$ goes to $i$ times the Poisson bracket in (2.3):

$$
\begin{aligned}
& \hat{u} \hat{u}-\hat{u} \hat{u} \rightarrow i \hat{u} r \hat{u}-i \hat{u} r \hat{u} \\
& \begin{array}{llllllll}
1 & 2 & 2 & 1 & 2 & 1 & 1 & 2
\end{array}
\end{aligned}
$$


So the task is to find $\hat{r}$ so that the commutation relations (4.3) agree with (1.2). Substituting (4.2) into (4.3), we get

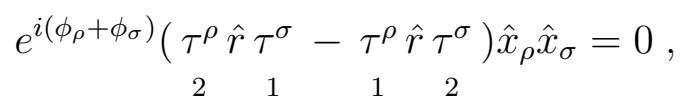

where the sum over indices is again assumed. We cannot equate coefficients of $\hat{x}_{\rho} \hat{x}_{\sigma}$ to zero because they are not all independent. Rather from (1.2) they are related by

$$
\hat{x}_{\rho} \hat{x}_{\sigma}=\frac{1}{2}\left(S_{\rho \sigma, \alpha \beta}+i \hat{E}_{\rho \sigma, \alpha \beta}\right) \hat{x}_{\alpha} \hat{x}_{\beta}
$$

where

$$
S_{\rho \sigma, \alpha \beta}=\delta_{\alpha \rho} \delta_{\beta \sigma}+\delta_{\alpha \sigma} \delta_{\beta \rho}
$$

and $\hat{E}_{\rho \sigma, \alpha \beta}$ is given in (1.3). Both $S_{\rho \sigma, \alpha \beta}$ and $\hat{E}_{\rho \sigma, \alpha \beta}$ are symmetric in the last two indices. (4.6) then says that all quadratic combinations of $\hat{x}_{\mu}$ can be expressed in terms of just the symmetric ones. If we substitute into (4.5) we can then equate coefficients of all the symmetric combinations of $\hat{x}_{\alpha} \hat{x}_{\beta}$ to zero. The result is a generalization of (2.5):

$$
\begin{aligned}
& \tau^{\rho} \hat{r} \tau^{\sigma}-\tau^{\rho} \hat{r} \tau^{\sigma}+\tau^{\sigma} \hat{r} \tau^{\rho}-\tau^{\sigma} \hat{r} \tau^{\rho} \\
& \begin{array}{llllllll}
2 & 1 & 1 & 2 & 2 & 1 & 1 & 2
\end{array} \\
& =-i e^{i\left(\phi_{\mu}+\phi_{\nu}-\phi_{\rho}-\phi_{\sigma}\right)} \hat{E}_{\mu \nu, \rho \sigma}\left(\tau^{\mu} \hat{r} \tau^{\nu}-\tau^{\mu} \hat{r} \tau^{\nu}\right)
\end{aligned}
$$

Up to an overall constant factor, it is solved by

$$
\hat{r}=\frac{1}{2}\left(\begin{array}{cccc}
e^{2 i\left(\phi_{0}-\phi_{2}\right)}+e^{2 i\left(\phi_{0}-\phi_{1}\right)} & 0 & 0 & e^{2 i\left(\phi_{0}-\phi_{2}\right)}-e^{2 i\left(\phi_{0}-\phi_{1}\right)} \\
0 & e^{2 i\left(\phi_{0}-\phi_{3}\right)}+1 & e^{2 i\left(\phi_{0}-\phi_{3}\right)}-1 & 0 \\
0 & e^{2 i\left(\phi_{0}-\phi_{3}\right)}-1 & e^{2 i\left(\phi_{0}-\phi_{3}\right)}+1 & 0 \\
e^{2 i\left(\phi_{0}-\phi_{2}\right)}-e^{2 i\left(\phi_{0}-\phi_{1}\right)} & 0 & 0 & e^{2 i\left(\phi_{0}-\phi_{2}\right)}+e^{2 i\left(\phi_{0}-\phi_{1}\right)}
\end{array}\right),
$$

or equivalently,

$$
\hat{r}=\mathbb{1}_{4 \times 4}+i \hat{\kappa}_{\mu} \tau^{\mu} \otimes \tau^{\mu}
$$

with a sum over $\mu$ and

$$
\begin{aligned}
\hat{\kappa}_{0} & =\frac{i}{4}\left(3-e^{2 i\left(\phi_{0}-\phi_{1}\right)}-e^{2 i\left(\phi_{0}-\phi_{2}\right)}-e^{2 i\left(\phi_{0}-\phi_{3}\right)}\right) \\
\hat{\kappa}_{1} & =-\frac{i}{4}\left(1+e^{2 i\left(\phi_{0}-\phi_{1}\right)}-e^{2 i\left(\phi_{0}-\phi_{2}\right)}-e^{2 i\left(\phi_{0}-\phi_{3}\right)}\right)
\end{aligned}
$$




$$
\begin{aligned}
& \hat{\kappa}_{2}=-\frac{i}{4}\left(1-e^{2 i\left(\phi_{0}-\phi_{1}\right)}+e^{2 i\left(\phi_{0}-\phi_{2}\right)}-e^{2 i\left(\phi_{0}-\phi_{3}\right)}\right) \\
& \hat{\kappa}_{3}=-\frac{i}{4}\left(1-e^{2 i\left(\phi_{0}-\phi_{1}\right)}-e^{2 i\left(\phi_{0}-\phi_{2}\right)}+e^{2 i\left(\phi_{0}-\phi_{3}\right)}\right)
\end{aligned}
$$

The expressions for $\hat{\kappa}_{i}, i=1,2,3$, reduce $\kappa_{i}$ to (2.7) in the limit of small angles $\phi_{\mu} \rightarrow \epsilon_{\mu}$, while $\hat{\kappa}_{0}$ is arbitrary in the limit.

The relations (4.3) are not invariant under Spin(4). Alternatively, we can try to define a deformation of $\operatorname{Spin}(4)$, parametrized by two nonsingular $2 \times 2$ matrices $\hat{g}$ and $\hat{h}$ with noncommuting matrix elements with an involution. The co-action on $\hat{u}$ is

$$
\hat{u} \rightarrow \hat{u}^{\prime}=\hat{g} \hat{u} \hat{h}^{-1}
$$

In order to preserve (4.1) we demand that $\hat{g}$ and $\hat{h}$ are unitary. Transformation (4.12) preserves the commutation relations (4.3) provided

$$
\begin{array}{cccc}
\hat{r} \hat{g} \hat{h}= & \hat{h} \hat{g} \hat{r} & {[\hat{g}, \hat{g}]=\left[\begin{array}{c}
\hat{h}, \hat{h} \\
12
\end{array} \quad \begin{array}{ccc}
1 & 1 & 2
\end{array}\right]=0}
\end{array}
$$

This is easily shown. Under the co-action, the left-hand-side of (4.3) transforms to

$$
\begin{aligned}
& \hat{u}^{\prime} \hat{r} \hat{u}^{\prime}=\hat{g} \hat{u} \hat{h}^{-1} \hat{r} \hat{g} \hat{u} \hat{h}^{-1} \\
& \begin{array}{llllllll}
1 & 2 & 1 & 1 & 1 & 2 & 2 & 2
\end{array} \\
& =\begin{array}{lllllll}
\hat{g} & \hat{u} & \hat{g} & \hat{r} & \hat{h}^{-1} & \hat{u} & \hat{h}^{-1} \\
1 & 1 & 2 & 1 & 2 & 2
\end{array}
\end{aligned}
$$

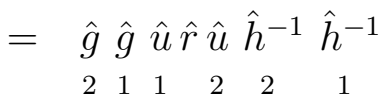

$$
\begin{aligned}
& =\hat{g} \hat{g} \hat{u} \hat{r} \hat{u} \hat{h}^{-1} \hat{h}^{-1} \\
& =\begin{array}{llllll}
\hat{g} & \hat{u} & \hat{g} & \hat{r} \hat{h}^{-1} & \hat{u} & \hat{h}^{-1} \\
2 & 2 & 1 & 2 & 1 & 1
\end{array} \\
& =\begin{array}{lllllll}
\hat{g} & \hat{u} & \hat{h}^{-1} & \hat{r} & \hat{g} & \hat{u} & \hat{h}^{-1} \\
2 & 2 & 2 & 1 & 1 & 1
\end{array}=\begin{array}{c}
\hat{u}^{\prime} \hat{r} \\
2
\end{array} \hat{u}^{\prime}
\end{aligned}
$$

In order for the algebra generated by $\hat{g}$ and $\hat{h}$ to be associative it is necessary for $\hat{r}$ to satisfy the quantum Yang-Baxter equations:

$$
\begin{aligned}
& \begin{array}{llllll}
\hat{r} & \hat{r} & \hat{r} & = & \hat{r} & \hat{r}
\end{array} \\
& \begin{array}{llllll}
12 & 13 & 23 & 23 & 13 & 12
\end{array}
\end{aligned}
$$


On the other hand, the quantum Yang-Baxter equations did not have to be satisfied for the algebra generated by $\hat{u}$ to be associative. Finally substitute (4.10) into (4.15). As in the infinitesimal cases, we get an identity only when two angles are equal and the third is zero. Hence the spinor-type transformations (4.12) correspond to symmetries only in the Connes-Landi case. For example, choose $\phi_{0}=\phi_{4}=0$ and $\phi_{1}=\phi_{2}=\phi / 2$. Then $\hat{r}$ simplifies to $\operatorname{diag}(q, 1,1, q)$, where $q=e^{-i \phi}$, and the condition (1.1) is equivalent to

$$
\operatorname{det}_{q} \hat{u} \equiv \hat{u}_{11} \hat{u}_{22}-q \hat{u}_{12} \hat{u}_{21}=1
$$

This condition is preserved under (4.12) provided $\operatorname{det} \hat{g} \operatorname{det} \hat{h}^{-1}=1$, after using (4.3) along with the commutation relations for matrix elements of $\hat{g}$ with $\hat{h}^{-1}$. It can be checked that both $\operatorname{det} \hat{g}$ and $\operatorname{det} \hat{h}^{-1}$ are Casimirs of the algebra and hence can be set to one. From (4.12) one can obtain the left co-action of the coordinates

$$
\hat{x}_{\mu} \rightarrow \hat{x}^{\prime}{ }_{\mu}=\hat{M}_{\mu \nu} \hat{x}_{\nu}
$$

with

$$
\hat{M}_{\mu \nu}(\hat{g}, \hat{h})=\frac{1}{2} e^{i\left(\phi_{\nu}-\phi_{\mu}\right)} \operatorname{Tr}\left(\tau_{\mu} \hat{g} \tau^{\nu} \hat{h}^{-1}\right)
$$

The commutation relations for $\hat{M}_{\mu \nu}$ are then determined from the commutation relations for matrix elements of $\hat{g}$ with $\hat{h}^{-1}$.

\section{Noncommutative hyperboloids}

The sphere of Connes and Dubois-Violette can be Wick rotated to Minkowski space. The result is a three-parameter family of "noncommutative hyperboloids". We can then repeat the previous analysis and search for quantum deformations of the Lorentz group which have noncommutative hyperboloids as homogeneous spaces. For simplicity, we only examine the first order system and write it in spinor notation. The result is that there is a Lie-Poisson action of a Lie-Poisson group acting on a one parameter subgroup of noncommutative hyperboloids, namely the Wick rotated version Connes-Landi spheres.

The Wick rotation of the Poisson structure (1.4) is

$$
\left\{x_{\mu}, x_{\nu}\right\}=E_{\mu \nu, \rho \sigma} x^{\rho} x^{\sigma}
$$

where $E_{\mu \nu, \rho \sigma}$ are again given in terms of three independent parameters by (1.5) and the indices of $x$ are raised and lowered by the Minkowski metric $\eta_{\mu \nu}=\operatorname{diag}(-1,1,1,1)$. $\eta_{\mu \nu} x^{\mu} x^{\nu}$ is a Casimir for the Poisson algebra and so we can restrict to a hyperboloid. In the quantized theory, the time component $x_{0}$ will be noncommuting for any nontrivial values of the parameters. 
The algebra can be re-expressed in terms of a hermitean matrix $x=x_{\mu} \sigma^{\mu}$, where $\sigma^{0}$ is the $2 \times 2$ identity matrix and $\sigma^{i}, i=1,2,3$, are the Pauli matrices. The Poisson brackets (5.1) can be written as

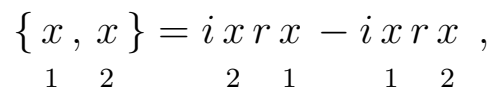

using the definition of $r$ in (2.6). The Casimir is now expressed as det $x$, the latter being invariant under $S L(2, C)$ transformations

$$
x \rightarrow x^{\prime}=s x s^{\dagger}, \quad s \in S L(2, C)
$$

The Poisson algebra is not preserved under this action, but if we can assign the following Poisson structure to $S L(2, C)$ :

$$
\{s, \bar{s}\}=-i[r, s \bar{s}] \quad\{s, s\}=\{\bar{s}, \bar{s}\}=0
$$

where $\bar{s}=s^{\dagger^{-1}}$, then the brackets (15.2) are invariant in the sense that (15.3) is a Poisson map. Once again for consistency we need to check the Jacobi identity, or equivalently the classical Yang-Baxter equations for $r$. But as before these conditions are only satisfied when all but one $\kappa_{i}$ vanish, and so we only get a consistent deformation of $S L(2, C)$ in the Connes-Landi limit. For a classification of consistent quantum deformations of $S L(2, C)$ see [10].

\section{Concluding Remarks}

Our search for quantum deformations of $\operatorname{Spin}(4)$ and $S O(4)$ for which the noncommutative three-spheres of Connes and Dubois-Violette are homogeneous spaces, and which have a smooth commutative limit in the Lie-Poisson sense, has yielded only the known symmetries of twisted Connes-Landi spheres. The question arises as to whether a more involved analysis can yield any other quantum deformations, possibly without a smooth commutative limit. Other possibilities which are currently under investigation would allow for more general Poisson structures on the group at the Poisson level, or commutation relations at the quantum level. In this regard, although the brackets (2.10), if they could have been consistently defined, would have assured that (1.6) is a Poisson map, other possibilities for the Poisson structure on $g$ and $h$ can be explored. For ex-

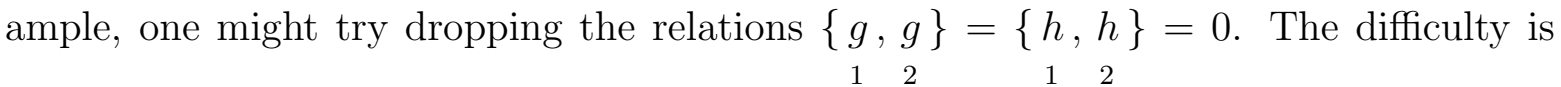


to make this consistent with the requirement that (1.6) be a Poisson map. Moreover, at the quantum level, one can consider generalizing the commutation relations (4.13),

possibly dropping $[\hat{g}, \hat{g}]=\left[\begin{array}{cc}\hat{h}, \hat{h} \\ 1\end{array}\right]=0$. The task then would be to find the analogue of (4.14). Finally, throughout this article we have insisted upon writing Poisson brackets and commutations relations in terms of an R-matrix. While this is an important case, other possibilities should be investigated for an exhaustive study.

\section{Acknowledgements}

We are very grateful to P. Aschieri, J.M. Gracia-Bondía and G. Landi for many useful discussions and correspondence. This work has been supported in part by the Progetto Nazionale di Interesse Nazionale Sintesi.

\section{REFERENCES}

[1] A. Connes, Noncommutative Geometry, Academic Press (1994); G. Landi, An introduction to Noncommutative Spaces and their Geometries, vol.51 of Lecture Notes in Physics. New Series M, Monographs (1997), (hep-th/9701078, J.M. Gracia-Bondia, J.C. Várilly, H.Figueroa, Elements of Noncommutative Geometry, Birkhäuser (2000); J.Madore, An introduction to noncommutative differential geometry and its physical applications, Lect.Notes London Math.Soc. 206 (1995) Cambridge University Press.

[2] L. Dabrowski, "The garden of quantum spheres", arXiv:math.qa/0212264; "The geometry of quantum spheres," arXiv:math.qa/0501240.

[3] A. Connes and M. Dubois-Violette, "Noncommutative finite-dimensional manifolds. I. Spherical manifolds and related examples," Commun. Math. Phys. 230, 539 (2002), arXiv:math.qa/0107070; "Moduli space and structure of noncommutative 3-spheres," Lett. Math. Phys. 66, 91 (2003) arXiv:math.qa/0308275.

[4] A. Connes and G. Landi, "Noncommutative manifolds: The instanton algebra and isospectral deformations," Commun. Math. Phys. 221 (2001) 141 arXiv:math.qa/0011194.

[5] J. C. Varilly, "Quantum symmetry groups of noncommutative spheres," Commun. Math. Phys. 221, 511 (2001) arXiv:math.qa/0102065; A. Sitarz, "Dynamical noncommutative spheres," Commun. Math. Phys. 241, 161 (2003) arXiv:math-ph/0112042. 
[6] P. Aschieri and F. Bonechi, "On the Noncommutative Geometry of Twisted Spheres," Lett. Math. Phys. 59, 133 (2002) arXiv:math.qa/0108136.

[7] A. Schirrmacher, "Multiparameter R matrices and its quantum groups," J. Phys. A 24, L1249 (1991).

[8] N. Reshetikhin, "Multiparameter Quantum Groups and Twisted Quasi-Traingular Hopf-Algebras," Lett. Math. Phys. 20, 331 (1990). A. Sudbery, "Consistent Multiparameter Quantization of Gl(N),"J. Phys. A.: Math. Gen. 23, L705 (1990). E. E. Demidov, Y. I. Manin, E. E. Mukhin and D. V. Zhdanovich, "Nonstandard Quantum Deformations Of $\mathrm{Gl}(\mathrm{N})$ And Constant Solutions Of The Yang-Baxter Equation," Prog. Theor. Phys. Suppl. 102, 203 (1990). D. B. Fairlie and C. K. Zachos, "Multiparameter Associative Generalizations Of Canonical Commutation Relations And Quantized Planes," Phys. Lett. B 256, 43 (1991).

[9] L. A. Takhtajan in Introduction to Quantum Groups and Integrable Models of Quantum Field Theories, M-L Ge and B-H Zhao eds, World Scientific, Singapore, 1989.

[10] S. Zakrzewski, "Poisson Poincaré groups," arXiv:hep-th/9412099 P. Podles and S. L. Woronowicz, "Quantum Deformation Of Lorentz Group," Commun. Math. Phys. 130 (1990) 381. J. A. de Azcarraga and F. Rodenas, "Deformed Minkowski spaces: classification and properties," J. Phys. A 29, 1215 (1996). 